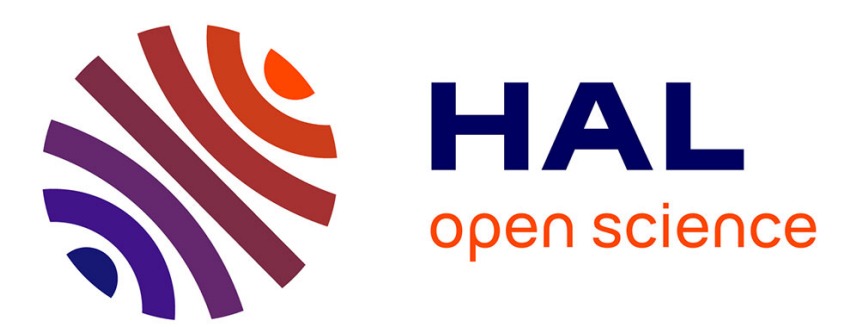

\title{
Energy minimization based resource scheduling for strict delay constrained wireless communications
}

\author{
Ibrahim Fawaz, Philippe Ciblat, Mireille Sarkiss
}

\section{To cite this version:}

Ibrahim Fawaz, Philippe Ciblat, Mireille Sarkiss. Energy minimization based resource scheduling for strict delay constrained wireless communications. 2017 IEEE Global Conference on Signal and Information Processing (GlobalSIP), Nov 2017, Montreal, Canada. 10.1109/GlobalSIP.2017.8308645 . cea-01888854

\section{HAL Id: cea-01888854 https://hal-cea.archives-ouvertes.fr/cea-01888854}

Submitted on 5 Oct 2018

HAL is a multi-disciplinary open access archive for the deposit and dissemination of scientific research documents, whether they are published or not. The documents may come from teaching and research institutions in France or abroad, or from public or private research centers.
L'archive ouverte pluridisciplinaire HAL, est destinée au dépôt et à la diffusion de documents scientifiques de niveau recherche, publiés ou non, émanant des établissements d'enseignement et de recherche français ou étrangers, des laboratoires publics ou privés. 


\title{
Energy minimization based Resource Scheduling for Strict Delay Constrained Wireless Communications
}

\author{
Ibrahim Fawaz ${ }^{1,2}$, Philippe Ciblat ${ }^{2}$, and Mireille Sarkiss ${ }^{1}$ \\ ${ }^{1}$ LIST, CEA, Communicating Systems Laboratory, F-91191 Gif-sur-Yvette, France \\ ${ }^{2}$ LTCI, Télécom ParisTech, Université Paris-Saclay, F-75013, Paris, France \\ Email: ibrahim.fawaz@cea.fr, philippe.ciblat@telecom-paristech.fr, mireille.sarkiss@cea.fr
}

\begin{abstract}
This paper investigates the energy consumption minimization for resource scheduling in a wireless communication. We propose to take into account a strict delay constraint for each queued packet rather than an average delay constraint, in addition to a buffer overflow constraint. The associated optimization problem can be modeled as Constraint Markov Decision Problem where the actions are the number of packets sent on the known channel at each slot. The optimal random policy is exhibited through the resolution of standard linear programming. We show the gain in energy is substantial compared to naive policy.
\end{abstract}

\section{INTRODUCTION}

Due to the now applications such Internet of Things and very-high data rate mobile cellular systems [1], the future wireless communication systems have to satisfy an unprecedented growth of the traffic and at the same time strong requirements on the latency, packet error rate, energy consumption, etc [2]. Focusing on resource scheduling algorithms, we propose to minimize the consumed energy by taking into account a new constraint related to the latency.

The context of energy efficient resource scheduling algorithms is huge and has been widely analyzed during the past decade. We hereafter briefly remind the main results related to our problem. In [3], the packet outage probability is minimized under average power and average delay constraints. Thanks to the so-called Little's Law, the average delay constraint has actually been converted into an average queue length constraint. Finally, based on Constrained Markov Decision Problem (CMDP) framework, they proposed a near-optimal deterministic policy that chooses the number of packets to send and the transmission power according to the queue state and the channel conditions. In [4], the average delay (or equivalently the average queue length) is minimized under average power constraint. Once again, the problem can be formulated through a CMDP. Using a Lagrangian approach enabling to transform the problem into an unconstrained MDP, they showed an optimal stationary random policy exists. In [5], the average power is minimized under average delay and packet outage probability constraints. They proposed a simple but sub-optimal deterministic policy that chooses the number

This work has received funding from the European Unions Horizon 2020 research and innovation programme under the Marie Skłodowska-Curie gran agreement No 675891. We also would like to acknowledge Dr. Romain Tajan (IMS, CNRS, ENSEIRB-Matmeca, Université de Bordeaux) for his fruitful advices. of packets to send according to the queue state whenever the channel magnitude exceeds a pre-fixed threshold. In [6], the average power is minimized under average delay constraint. Like [4], they converted the CMDP into an unconstrained MDP in order to exhibit the optimal stationary policy that eventually chooses to transmit more packets when the buffer occupancy increases. In [7], the average power is minimized under average delay constraint. Unlike [6], they proposed an online implementation of the policy characterization. In addition, their implementation was adapted to the uncertainty of the underlying Markov chain transition matrix and of the packet arrival distribution, by using virtual states called postdecision states and leading to modifications of the Relative Value Iteration algorithm. Whereas above contributions have proposed efficient solutions when average delay is the relevant metric, they are not adapted yet to many future applications requiring stringent delay. Therefore, it is of interest to replace the average delay constraint with a strict delay constraint, i.e., the packets should be scheduled before a deadline. For instance, in [8], bit scheduling for one packet with strict delay and no random packet arrival is performed. In [9], a predefined set of packets have to be sent under a strict deadline for any active user.

Our main contribution is to find an optimal stationary policy minimizing the average power under strict delay constraint with random packet arrival. We formulate the problem as a CMDP, solve it using Linear Programming (LP), and compare the optimal policy to a naive one based on best effort strategy.

The remainder of the paper is organized as follows. In Section II, we describe the system model. In Section III, we formulate the optimization problem as a CMDP. In Section IV, we solve the CMDP using LP. Numerical results are provided and analyzed in Section V. Finally, concluding remarks and future works are drawn in Section VI.

\section{SYSTEM MODEL}

We consider a slotted wireless communication over block flat-fading channel. The duration of each slot is $T_{s}$. At slot $n$, the channel is constant with complex-valued amplitude $h_{n}$. We define the channel gain as follows $x_{n}=\left|h_{n}\right|^{2}$. We assume $\left\{x_{n}\right\}_{n}$ is an i.i.d. process following a distribution into a finite set $\mathcal{X}$. 
The transmitter has a buffer of size $B$ packets. The packets (coming from the upper layer) are of size $L$ bits. They are always queued in the buffer before the transmitter decides to transmit them. The transmitter receives $a_{n}$ packets from the upper layer between slot $n-1$ and $n$. We assume $\left\{a_{n}\right\}_{n}$ is an i.i.d. process following a Poisson distribution with an average arrival rate $\gamma$, i.e., the probability to generate $a$ packets at time $n$ is

$$
p_{A}\left(a_{n}=a\right)=e^{-\gamma} \cdot \frac{\gamma^{a}}{a !} .
$$

At the beginning of slot $n$, the queue length in the buffer is denoted by $q_{n}$ packets. Notice that $q_{n} \in\{0, \ldots, B\}$. We also denoted by $u_{n}$ the number of transmit packets during slot $n$.

A packet is discarded from the buffer

- if there is a buffer overflow, i.e., if the number of packets in the queue exceeds the buffer size. In that case, we discard the arrival packets in overflow; or

- if there is a delay no-fulfillment, i.e., it stays in the queue more than $K_{0}$ slots.

While the buffer overflow can be described using $q_{n}$, other variables (indicating more precisely the buffer configuration) are needed for analyzing the delay no-fulfillment. Consequently, we denoted by $k_{i}(n)$ the time (counting in slot) spent in the buffer by the $i$-th packet at time $n$ as shown in Fig. 1 . By convention, $k_{i}(n)=-1$ means there is no $i$-th packet. So $k_{i}(n) \in\left\{-1, \ldots, K_{0}\right\}$ for any $i$ and $k$. Moreover, according to Fig. 1 , we get $\forall i \leqslant j, k_{j}(n) \leqslant k_{i}(n)$.

Buffer of $B$ packets (ordered from the oldest to the newest) \begin{tabular}{|c|c|c|c|c|c|}
\hline$k_{1}(n)$ & $\cdots$ & $k_{q_{n}}(n)$ & -1 & $\cdots$ & -1 \\
\hline & \multicolumn{2}{|c|}{ empty area }
\end{tabular}

Fig. 1: Buffer configuration at slot $n$.

For a channel with a gain $x$, a power spectral density $N_{0}$, and a signal bandwidth $W$, the power required to transmit $u$ packets within a slot of duration $T_{s}$ can be expressed as follows [7]:

$$
P(x, u)=\frac{W N_{0}}{x}\left(2^{\frac{u L}{W T_{s}}}-1\right) .
$$

During slot $n$, the transmitter sends the $u_{n}$ oldest packets through the channel of gain $x_{n}$ with a power $P\left(x_{n}, u_{n}\right)$. We assume Channel State Information at the Transmitter (CSIT).

\section{Problem Formulation}

Our objective now is to find the optimal policy minimizing the power consumption under strict delay outage and buffer overflow outage constraints. The policy is a sequence of actions that specifies at each slot the number of packets $u$ to be scheduled based on the history of the system and the past actions. A powerful tool for such a problem may be the socalled CMDP. We show, in this section, that our problem boils down CMDP by defining appropriate states, actions, reward, and constraints [10].

\section{A. State Space}

The state space $\mathcal{S}$ is the set of $\mathbf{s}=(\mathbf{k}, x)$ where

- $\mathbf{k}=\left[k_{1}, \cdots, k_{B}\right]$ is the vector indicating the age of each packet in the buffer, and

- $x$ is the channel gain.

Notice that in the state-of-the-art [3]-[7], the states were different since $\mathrm{k}$ were replaced with the queue length $q$. Here the knowledge of $q$ is not enough to describe our buffer due the strict delay constraint. Actually, $q$ is even unnecessary when $\mathbf{k}$ is given since

$$
q_{n}=\max \left\{i \mid k_{i}(n) \geqslant 0\right\} .
$$

The state space is finite, and the total number of possible states is $|\mathcal{S}|$ which is upper-bounded by $\left(K_{0}+2\right)^{B} \cdot|\mathcal{X}|$. As the packets are queued in an increasing order of time spent in the buffer, we always have $k_{1}(n) \geq k_{2}(n) \geq \cdots \geq k_{q_{n}}(n)$ which dramatically reduces the state space compared to the upper-bound. For instance, if we consider $B=5, K_{0}=2$, and $|\mathcal{X}|=3$, the upper-bound is 3072 while, by removing the impossible combination of components in $\mathbf{k}$, our system only considers 168 states.

\section{B. Action Space}

The action space $\mathcal{U}$ denotes the number of packets $u$ that the transmitter will send through the channel. Let $U_{0}$ be the maximum number of packets that can be transmitted during a slot. $U_{0}$ is obtained by using Eq. (1) with the maximum available power $P_{\max }$ and the best channel coefficient $x_{\max }=$ $\max _{x \in \mathcal{X}} x$. Finally, the action space is finite and the number of actions is $|\mathcal{U}|=U_{0}+1$.

\section{Markov Decision Process}

During slot $n, w_{n}=\max \left(u_{n}, m_{n}\right)$ packets leave the buffer (either transmitted or discarded), where $u_{n}$ is the number of packets transmitted and $m_{n}$ is the number of packets with delay $K_{0}$ slots in the buffer. The age of the remaining packets in the buffer is incremented by 1 . Moreover, $a_{n+1}$ new packets arrive to the buffer with age 0 . Therefore the update for vector $\mathbf{k}$ from slot $n$ to slot $n+1$ can be done as follows:

$$
\left\{\begin{array}{l}
k_{1}(n+1)=k_{w_{n}+1}(n)+1 \\
\vdots \\
k_{q_{n}-w_{n}}(n+1)=k_{q_{n}}(n)+1 \\
k_{q_{n}-w_{n}+1}(n+1)=0 \\
\vdots \\
k_{q_{n}-w_{n}+a_{n+1}}(n+1)=0 \\
k_{q_{n}-w_{n}+a_{n+1}+1}(n+1)=\cdots=k_{B}(n+1)=-1 .
\end{array}\right.
$$

Notice that when $q_{n}-w_{n}+a_{n+1}$ is larger than $B$, the buffer accepts a part of the incoming packets and discards the remaining $\left(q_{n}-w_{n}+a_{n+1}-B\right)$ incoming packets. In that case, the buffer is full $\left(q_{n}-w_{n}+a_{n+1}=B\right)$ and the last equation 
in (3) is omitted. We thus remark that $\mathbf{k}_{n+1}$ only depends on previous state $\mathbf{k}_{n}$, action $u_{n}$ and external perturbation $a_{n+1}$.

Therefore we can define $p\left(\mathbf{s}^{\prime} \mid \mathbf{s}, u\right)$ as the transition probability to fall in the state $\mathbf{s}^{\prime}=\left(\mathbf{k}^{\prime}, x^{\prime}\right)$ after taking action $u$ in the current state $\mathbf{s}=(\mathbf{k}, x)$. Assuming that the buffer and channel states are independent and channel states are not correlated, the transition probability satisfies the following equation:

$$
p\left(\mathbf{s}^{\prime} \mid \mathbf{s}, u\right)=p\left(\mathbf{k}^{\prime} \mid \mathbf{k}, u\right) \cdot p\left(x^{\prime}\right)
$$

where $p\left(x^{\prime}\right)$ is the distribution of the channel states, and where $p\left(\mathbf{k}^{\prime} \mid \mathbf{k}, u\right)$ indicates the probability transitions between buffer states. After tedious but simple derivations, we obtain:

If $u>q$ or $k_{i}^{\prime}>k_{i}+1$ or $q^{\prime}<q-w$,

$p\left(k_{i}^{\prime} \mid k_{i}, u\right)=0$ else

If $k_{i}^{\prime} \neq k_{i+u}+1$ and $k_{i+u} \neq-1$,

$p\left(k_{i}^{\prime} \mid k_{i}, u\right)=0$ else

If $k_{i}^{\prime}>0$ and $k_{i+u}=-1$,

$p\left(k_{i}^{\prime} \mid k_{i}, u\right)=0$ else

If $q=B$ and $u \neq 0$ and $k_{i}^{\prime}>0, \forall i \in\{q-w+1, \ldots, B\}$,

$p\left(k_{i}^{\prime} \mid k_{i}, u\right)=0$ else

If $q^{\prime}<B$,

$p\left(k_{i}^{\prime} \mid k_{i}, u\right)=e^{-\gamma} \times \frac{\gamma^{a}}{a !}$ else

If $q^{\prime}=B$,

$p\left(k_{i}^{\prime} \mid k_{i}, u\right)=1-Q(B-q+w, \gamma)$.

end,

where $Q$ is the regularized gamma function.

\section{Constrained Markov Decision Problem}

To complete the description of the CMDP, it remains to define the cost and constraint functions. In the context of infinite horizon CMDP, we consider time-averaged cost, where at a given time slot $n \in\{0, \cdots, N\}$, the system state is denoted by $\mathbf{s}_{n}=\left(\mathbf{k}_{n}, x_{n}\right)$ and $\mu\left(\mathbf{s}_{n}\right)=u_{n}$ is the action deciding the number of packets to be transmitted. We would like to find the optimal policy $\mu^{\star}$ minimizing the average consumed power given by the following equation:

$$
\bar{P}^{\mu}=\lim _{N \rightarrow+\infty} \frac{1}{N} \mathbb{E}^{\mu}\left[\sum_{n=1}^{N} P\left(x_{n}, u_{n}\right)\right]
$$

where $\mathbb{E}$ is the expectation with respect to the policy $\mu$ and where $P\left(x_{n}, u_{n}\right)$ is the instantaneous power cost when action $u_{n}$ is performed at state $\mathbf{s}_{n}$ and can be derived using Eq. (1)

At a given slot $n$, when the system state is $\mathbf{s}_{n}$ and the performed action is $u_{n}$, the probability to discard packets due to delay no-fulfillment is given by the following equation

$$
\varepsilon_{d}\left(\mathbf{s}_{n}, u_{n}\right)= \begin{cases}0 & \text { if } m_{n}=0 \text { or } m_{n} \leqslant u_{n} \\ 1 & \text { else. }\end{cases}
$$

We thus define the average delay no-fulfillment outage probability as:

$$
\bar{\varepsilon}_{d}^{\mu}=\lim _{N \rightarrow+\infty} \frac{1}{N} \mathbb{E}^{\mu}\left[\sum_{n=1}^{N} \varepsilon_{d}\left(\mathbf{s}_{n}, u_{n}\right)\right] .
$$

At a given slot $n$, when the system state is $\mathbf{s}_{n}$ and the performed action is $u_{n}$, the buffer overflow occurs when $q_{n}-$
$w_{n}+a_{n+1}>B$. The probability of overflow thus is obtained as follows

$$
\begin{aligned}
\varepsilon_{o}\left(\mathbf{s}_{n}, u_{n}\right) & =\sum_{a=B-q_{n}+w_{n}+1}^{+\infty} e^{-\gamma} \cdot \frac{\gamma^{a}}{a !} \\
& =1-Q\left(B-q_{n}+w_{n}+1, \gamma\right) .
\end{aligned}
$$

Thus, we define the average buffer overflow outage probability as:

$$
\bar{\varepsilon}_{o}^{\mu}=\lim _{N \rightarrow+\infty} \frac{1}{N} \mathbb{E}^{\mu}\left[\sum_{n=1}^{N} \varepsilon_{o}\left(\mathbf{s}_{n}, u_{n}\right)\right]
$$

Finally, our optimization problem, which boils down to CMDP, states as follows

Problem 1:

$$
\begin{aligned}
\mu^{\star}=\arg \min _{\mu} & \bar{P}^{\mu} \\
\text { s.t. } & \bar{\varepsilon}_{d}^{\mu} \leqslant D_{\text {out }} \\
& \bar{\varepsilon}_{o}^{\mu} \leqslant O_{\text {out }}
\end{aligned}
$$

where $D_{\text {out }}$ and $O_{\text {out }}$ are the pre-defined thresholds for the delay and overflow outage probabilities respectively.

\section{Problem Resolution}

As Problem 1 is CMDP, it can be solved using standard LP techniques to find the optimal offline randomized policy, which consists in a probabilistic mapping from the state space $\mathcal{S}$ to the action space $\mathcal{U}$. To exhibit the optimal policy, we are looking for the so-called occupation measure defined as a probability measure over the set of state-action pairs and denoted by $\rho^{\mu}(\mathbf{s}, u)$, The average cost function (here, the power) as well as the average outage probabilities constraints (here, the delay and the overflow) can be expressed with respect to $\rho^{\mu}(\mathbf{s}, u)$. In addition, it is shown in [10], that the optimal policy is stationary, which means it does not depend on the time at which the decision is made. Therefore Problem 1 can be formulated as the following LP problem.

Problem 2:

$$
\begin{aligned}
\rho^{\star}=\arg \min _{\rho} & \sum_{\mathbf{s} \in \mathcal{S}, u \in \mathcal{U}(\mathbf{s})} \rho^{\mu}(\mathbf{s}, u) P(x, u) \\
& \sum_{\mathbf{s} \in \mathcal{S}, u \in \mathcal{U}(\mathbf{s})} \rho^{\mu}(\mathbf{s}, u) \varepsilon_{d}(\mathbf{s}, u) \leqslant D_{\text {out }} \\
& \sum_{\mathbf{s} \in \mathcal{S}, u \in \mathcal{U}(\mathbf{s})} \rho^{\mu}(\mathbf{s}, u) \varepsilon_{o}(\mathbf{s}, u) \leqslant O_{\text {out }} \\
& \sum_{\mathbf{s} \in \mathcal{S}, u \in \mathcal{U}(\mathbf{s})} \rho^{\mu}(\mathbf{s}, u)=1 \\
& \sum_{\mathbf{s}^{\prime} \in \mathcal{S}, u \in \mathcal{U}(\mathbf{s})} \rho^{\mu}\left(\mathbf{s}^{\prime}, u\right) p\left(\mathbf{s} \mid \mathbf{s}^{\prime}, u\right)=1, \forall \mathbf{s} \in \mathcal{S}
\end{aligned}
$$

where $\mathcal{U}(\mathbf{s})$ is the set of possible actions when the system is in state $\mathbf{s}$.

Notice that Eq. (17) comes from the Markov property of the process $\left(\mathbf{s}_{n}, u_{n}\right)$. 
The optimal stationary policy $\mu^{\star}$ is then obtained from $\rho^{*}$ according to the following equation

$$
\mu^{\star}(u \mid \mathbf{s})=\frac{\rho^{*}(\mathbf{s}, u)}{\sum_{u^{\prime} \in \mathcal{U}(\mathbf{s})} \rho^{*}\left(\mathbf{s}, u^{\prime}\right)}
$$

whenever the denominator is non-zero. When it is zero, $\mu^{\star}(. \mid \mathbf{s})$ is chosen to be an arbitrary probability measure over $\mathcal{U}(\mathrm{s})$ [10]. Such states are known to be transient state and the taken action does not affect the system in the long-term. Moreover we have remarked that the obtained optimal policy chooses at most 3 (instead of $U_{0}$ ) possible actions randomly for each state since there are 2 constraints as already evoked in [10]. Actually in the majority of states, the optimal policy chooses even a single action in a deterministic way.

\section{NumericAl RESUlts}

We evaluate numerically the optimal policy obtained for the resolution of Problem 2. We consider a system as described in Section II with the following characteristics: the slot duration is $T_{s}=1 \mathrm{~ms}$, the channel states $x$ takes 3 possible values from the finite set $\mathcal{X}=\{-5.41,-1.59,3.18\} \mathrm{dB}$ with respective probabilities $0.63,0.32$, and 0.05 . The noise power spectral density is $N_{0}=-87 \mathrm{dBm} / \mathrm{Hz}$ and the allocated bandwidth is $W=5 \mathrm{MHz}$. We simulate i.i.d arrivals following a Poisson distribution with mean $\gamma=2$ packets per slot. We assume that packets are of equal size $L=5000$ bits, and the buffer is of size $B=5$ packets. The maximum delay is $K_{0}=2$ (i.e., in absolute time $K_{0} T_{s}=2 \mathrm{~ms}$ ). The maximum available power at the transmitter is $P_{\max }=2 \mathrm{~mW}$. According to Eq. (1), this system allows to transmit up to 8 packets per slot. Due to the buffer size, we fix $U_{0}=5$ packets per slot.

Fig. 2 shows the convergence behavior of the average power for various delay outage probabilities where the overflow outage probability $O_{\text {out }}$ is fixed 0.4 . One can notice that as the delay constraint becomes tighter, the average consumed power significantly increases. Indeed, when the delay required by the application is stricter, the system is forced to send more packets even if the channel is in a bad state.

Fig. 3 compares the average consumed power versus the delay outage probability for different overflow outage probabilities. Moreover we compare the optimal policy with a naive one which forces the transmitter to flush the buffer, i.e., send all the packets in the buffer whatever the channel conditions. Notice that the system characteristics have been chosen such that the naive policy satisfies the overflow outage probability constraint. The delay outage probability constraint is always satisfied by the naive policy since the packets do not stay in the buffer. As one can observe, our policy gives strongly better performance in terms of power consumption as it adapts its transmission rate according to the channel conditions while satisfying the requirements of the application. The overflow constraint is inactive when $O_{\text {out }}=0.4$ (blue line), because satisfying the delay constraint is more crucial. In contrast, when $O_{\text {out }}=0.05$ (pink line), the delay outage probability is inactive. In general, requiring a stronger overflow constraint

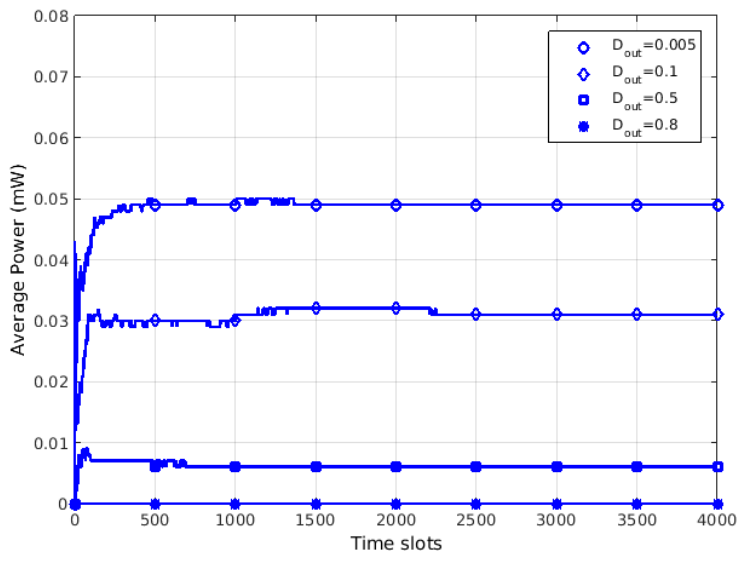

Fig. 2: Convergence of average power for various delay outage probability constraints.

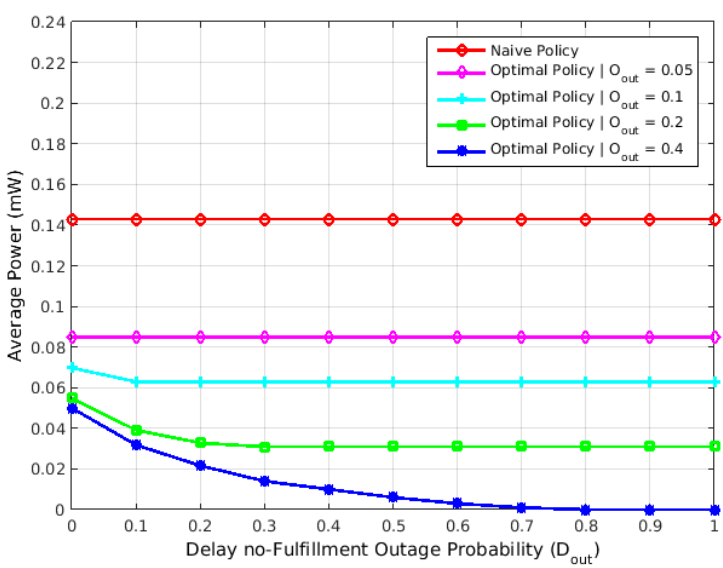

Fig. 3: Average power versus delay outage probability for different policies.

increases the energy consumption. Notice that after a certain value of $D_{\text {out }}$, the overflow constraint becomes more critical making the delay constraint inactive leading to a error floor.

\section{CONCLUSION AND Future WORKS}

We have addressed strict delay constrained scheduling problem. We have solved the power-efficient optimization problem using CMDP framework and so linear programming techniques. The policy adjusts the number of transmitted packets according to the channel conditions, such that the power consumed is minimized while maintaining the delay and overflow outage probabilities below pre-defined thresholds given by the application. As an extension to this work, we aim to include offloading capabilities to our system, and adapt it to the sustainable next generation 5G mobile networks by investigating an energy harvesting communication system where the terminal can scavenge energy from surrounding environment to power their communication and computing. 


\section{REFERENCES}

[1] Cisco, "Visual Networking Index," White Paper, February 2015. Available online: www.cisco.com.

[2] GSMA Intelligence, "Understanding 5G: Perspectives on future technological advancements in mobile," White Paper, December 2014. Available online: www.gsmaintelligence.com.

[3] D. Rajan, A. Sabharwal and B. Aazhang, "Transmission Policies for Bursty Traffic Sources on Wireless Channels," Conference on Information Sciences and Systems (CISS), March 2001.

[4] M. Goyal, A. Kumar and V. Sharma, "Power Constrained and Delay Optimal Policies for Scheduling Transmission over a Fading Channel," IEEE International Conference on Computer Communications (INFOCOM), March 2003.

[5] H. Wang and N. B. Mandayam, "A Simple Packet-Transmission Scheme for Wireless Data Over Fading Channels," IEEE Transactions on Communications, vol. 52, no. 7, pp. 1055-1059, July 2004.

[6] D. V. Djonin and V. Krishnamurthy, "Structural Results on the Optimal Transmission Scheduling Policies and Costs for Correlated Sources and Channels," IEEE Conference on Decision and Control (CDC), December 2005.

[7] N. Salodkar, A. Bhorkar, A. Karandikar and V. S. Borkar, "An Online Learning Algorithm for Energy Efficient Delay Constrained Scheduling over a Fading Channel," IEEE Journal on Selected Areas in Communications, vol. 26, no. 4, pp. 732-742, May 2008.

[8] L. Juyul and N. Jindal, "Energy-efficient scheduling of delay constraint traffic over fading channels," IEEE Transactions on Wireless Communications, vol. 8, no. 4, pp. 1866-1875, April 2009.

[9] M. De Mari and T. Quek, "Energy-efficient proactive scheduling in ultra dense networks," IEEE International Conference on Communications (ICC), May 2017

[10] E. Altman, "Constrained Markov Decision Processes,", Chapman \& Hall, 2004. 